\section{Estimation of Ascorbic Acid (Vitamin C) by Titration}

THE chemical determination of ascorbic acid is based upon the high reducing capacity shown by this substance with different organic compounds. One of the best known methods based upon this property is the titration of ascorbic acid with $2: 6$ dichlorphenol indophenol ${ }^{1}$, after the removal of interfering substances with mercuric acetate. Others ${ }^{2}$ have found that ascorbic acid reacts with methylene blue, and this property was made use of for its estimation. This method appears to be the most satisfactory, because the presence of other reducing substances does not interfere with the reactions linked to certain hydrogen ion concentrations. It has, however, certain disadvantages inherent to its colorimetrical nature. It is on account of this circumstance that I have tried to modify this method of estimation and thus to eliminate possible sources of error.

Several investigators having found that trichloroacetic acid seriously interferes with the stability of ascorbic acid, I have used for its extraction-according to Fujita's method ${ }^{3}$-metaphosphoric acid, which does not react either with methylene blue or with ascorbic acid. Martini and Bonsignore claim that the reaction of methylene blue is specific for vitamin $\mathrm{C}$ after a previous treatment of the solution with sodium citrate, sodium bicarbonate and thiosulphate in adequate quantities. These findings have been confirmed. But I have also taken into account the fact that methylene blue can be decolorized by different inorganic compounds. It seemed therefore logical to suppose that after treatment with general agents and exposure to light, the methylene blue remaining might be titrated with the aid of an adequate reducing compound. I have found that titanium trichloride (Merck) is a suitable substance for such purposes. However, as titanium trichloride reacts in certain concentrations with methylene blue (in the same manner as it does with other azo dyestuffs), it was necessary to prepare it in a suitable dilution in order to neutralize its action. I have found that a dilution of 5 in 1,000 answers this purpose. Below this limit no reaction can be observed.

It is important that the titanium trichloride solu. tion should always be prepared freshly and determined against a standard methylene blue solution. The methylene blue solution (Höchst, med.) must be pure and kept cool, and the determination must be made rapidly. It is known that solutions containing ascorbic acid will decolourize the methylene blue added to it. In consequence, a standard methyl. ene blue solution is added to the solution to be examined for its ascorbic acid content, until, after exposure to intense sunlight or to a 300 watt Philip's lamp, the colour of the solution to be titrated changes no more (time of irradiation $30-50 \mathrm{sec}$.). The quantity of methylene blue added to the solution being known, and knowing also the quantity of titanium trichloride necessary to decolourize the dyestuff remaining, these two data are sufficient to calculate the ascorbic acid content of the medium under investigation. Thus, for example, $1 \mathrm{ml}$. of methylene blue being equivalent to $0.047 \mathrm{mgm}$. ascorbic acid and the titanium trichloride content being proportional to the methylene blue concentration, it is easy to calculate the ascorbic acid content of the substance investigated from the quantity of titanium trichloride used to reduce the methylene blue not reacting with ascorbic acid.
Should it be necessary to estimate the oxidized ascorbic acid content of the substance in question, the mercuric acetate method ${ }^{4}$ above can be recommended; but care must be taken to remove any hydrogen sulphide, as it reacts with methylene blue. It is therefore absolutely necessary to carry out the reaction in a stream of carbon dioxide. The solution must be slightly acid. This modified method can be used for the estimation of the ascorbic acid content of both vegetable and animal tissues.

Hungarian Biological Research Institute,

IMRE GÁ. Tihany, Hungary.

${ }^{1} \mathrm{H}$. Tillmans, Z. Cnter8. Lebemen., 63, 1; 63, 241 (1932). E. Martini and A. Bonsignore, Biochem. Z., 273, 170 (1934).

(1935). Fujita, D. and Miyata Iwatake, Biochem. Z., 277, 293, 296

Mrthons for the determination of ascorbic acid have recently boen much discussed. Tillmans' method, as modified by Harris and Ray ${ }^{1}$, is generally accepted as more specific and simpler than the other methods proposed.

However, the rapid oxidation of ascorbic acid necessitates carrying out the titration in as short a time as possible, if consistent results are to be obtained. We have now found that by cooling the ascorbic acid solution to $0^{\circ} \mathrm{C}$., and by carrying out the titration at this temperature, it is possible to slow down very appreciably the oxidation, so that a more constant end point is obtained, and time is no longer such a fundamental factor in the results.

A more detailed account of this slight modification of Harris and Ray's method will appear elsewhere, but we think this brief statement of the facts may be of use to those interested in these problems.

Henri Cheftel.

Marie-Louise Pigeaud.

Laboratoire de Recherches Biologiques,

J. J. Carnaud et Forges de Basse-Indre,

71 Avenue Edouard Vaillant,

Boulogne-Billancourt.

${ }^{1}$ Harris and Ray, Biochem. J., 27, 303 (1933).

\section{Comparison of Mineral and Biological Potassium in Diet Experiments}

DURING the past eighteen months, we have been investigating, by means of diet experiments on mice, the question whether potassium of mineral origin is equivalent to that of biological origin, in view of the possibility that the isotopic constitution may be different. Brewer ${ }^{1}$ has recently reported that some of the samples of vegetable potassium tested differed slightly from mineral potassium.

The food utilized consisted of water-extracted maize-meal and casein, yeast extract (rendered potassium poor), cod liver oil, water-extracted agaragar and a salt mixture. The potassium was added to the salt mixture as a chloride. The 'biological' potassium chloride was prepared from the dry residue of the united extracts of the maize, casein and agar-agar, while $\mathrm{KCl}(A R)$ was used as a source of 'mineral' potassium. Four groups of 10 or 12 mice were used in each experiment, two serving for testing any difference in the two types of potassium and two serving as controls.

The results so far indicate that a difference in action of the two kinds of potassium exists, at least under certain conditions; but that the matter, 